This item was submitted to Loughborough's Research Repository by the author.

Items in Figshare are protected by copyright, with all rights reserved, unless otherwise indicated.

\title{
Field emission and lifetime of microcavity plasma
}

PLEASE CITE THE PUBLISHED VERSION

PUBLISHER

(C) American Institute of Physics

VERSION

VoR (Version of Record)

LICENCE

CC BY-NC-ND 4.0

REPOSITORY RECORD

Kim, G.J., Felipe Iza, and Jae Koo Lee. 2019. "Field Emission and Lifetime of Microcavity Plasma". figshare. https://hdl.handle.net/2134/5344. 
This item was submitted to Loughborough's Institutional Repository (https://dspace.lboro.ac.uk/) by the author and is made available under the following Creative Commons Licence conditions.

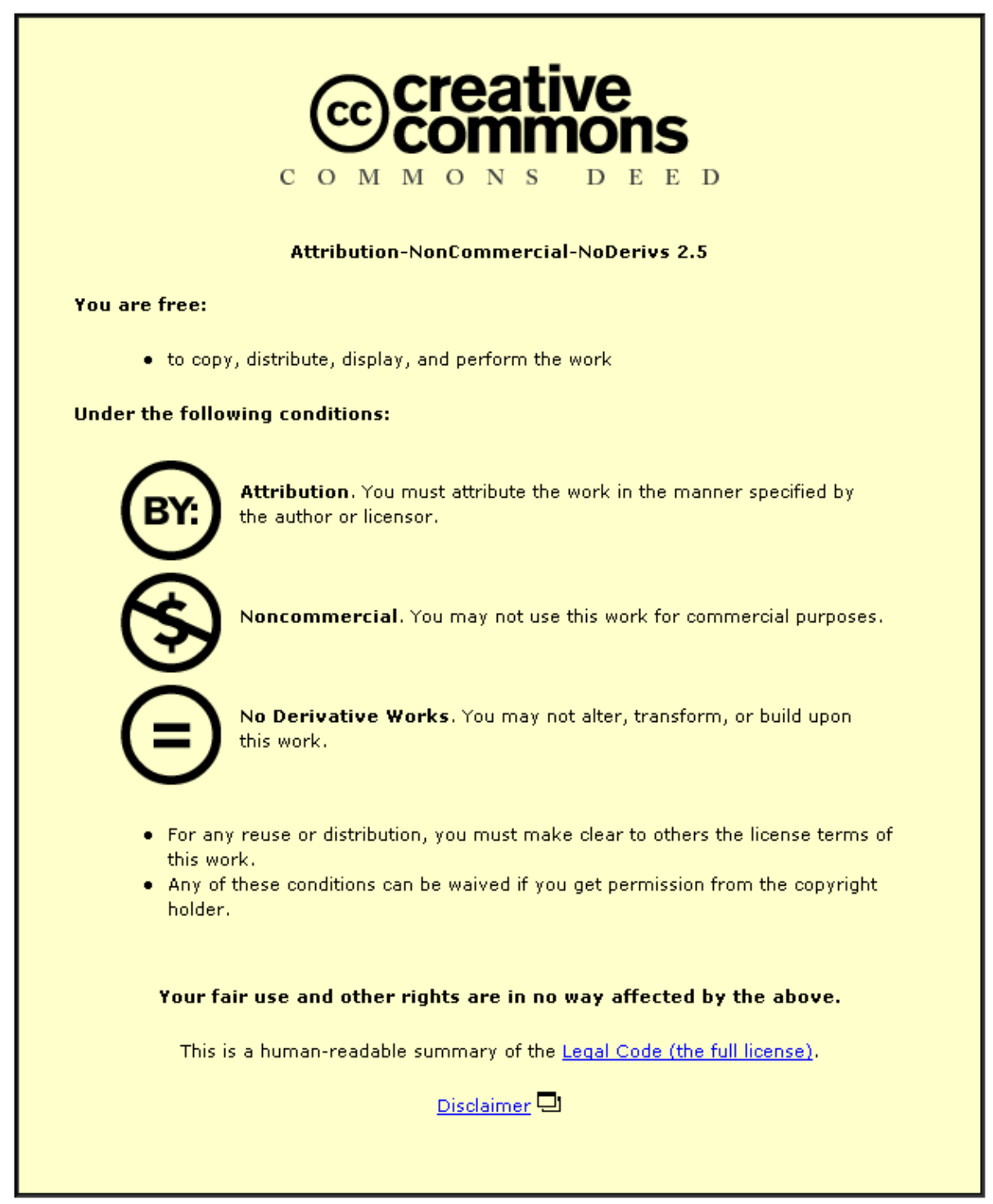

For the full text of this licence, please go to: http://creativecommons.org/licenses/by-nc-nd/2.5/ 


\title{
Field emission and lifetime of microcavity plasma
}

\author{
G. J. Kim, ${ }^{1}$ F. Iza, ${ }^{2}$ and J. K. Lee ${ }^{1}$ \\ ${ }^{1}$ Department of Electronic and Electrical Engineering, Pohang University of Science and Technology, \\ Pohang 790-784, Republic of Korea \\ ${ }^{2}$ Department of Electronic and Electrical Engineering, Loughborough University, Leicestershire LE11-3TU, \\ United Kingdom
}

(Received 14 August 2008; accepted 19 December 2008; published online 30 January 2009)

\begin{abstract}
Microplasmas with cylindrical hollow cathode have been studied by means of two-dimensional particle-in-cell/Monte-Carlo collision (PIC/MCC) simulations. For a given input power, the onset of field emission from the cathode surface caused by the strong electric field generated in these discharges leads to a reduction of the discharge voltage and an increase in plasma density. The plasma density profile can be strongly influenced by localized enhancements of the electric field, which in turn will affect the erosion profile of the cathode. The cathode erosion profile is predicted in this work by combining the ion kinetic information obtained from the PIC/MCC simulation with the sputtering yield computed using SRIM [J. F. Ziegler, J. P. Biersack, and M. D. Ziegler, SRIM: The Stopping and Range of Ions in Matter (Lulu, Chester, 2008)]. The entrance of the cathode and the center region are the areas most susceptible to ion-induced damage. The lifetime of the device, however, can be extended by operating the device at high pressure and by reducing the operating voltage by means of field emission and/or additional electron emitting processes from the cathode. (C) 2009 American Institute of Physics. [DOI: 10.1063/1.3068745]
\end{abstract}

\section{INTRODUCTION}

Microplasmas with cylindrical hollow cathode have received a lot of attention because of their capability of generating high density plasma, effective generation of energetic electrons, nonequilibrium characteristics and simple design. ${ }^{1,2}$ These features have prompted the investigation of microdischarges in many applications including surface processing, ${ }^{3,4}$ UV light sources, ${ }^{5}$ analytical chemistry, ${ }^{6}$ switches, ${ }^{7}$ and biomedical applications. ${ }^{8}$ In the last decade, discharges with cylindrical hollow cathode evolved into atmospheric pressure operation by reducing the cathode diameter to $10 \mu \mathrm{m},{ }^{9,10}$ eliminating the need for vacuum pumps and further contributing to the popularity of these devices. ${ }^{11}$ Hollow cathode discharges have been studied experimentally, ${ }^{12}$ theoretically, ${ }^{13}$ and computationally. ${ }^{14-16}$ Computer simulations offer a valuable tool to study microdischarges since they provide information that is hard to obtain experimentally due to the reduced dimensions of the discharges. ${ }^{15,17}$ In agreement with indirect evidence obtained from optical emission spectroscopy, ${ }^{2,10}$ recent simulation results ${ }^{15}$ indicate that as in large scale devices operated at lower pressure, the electron energy distribution function in microhollow cathodes also presents a non-Maxwellian distribution and a high energy tail.

The main drawback of discharges having cylindrical hollow cathode is their limited lifetime. To analyze the erosion profile caused by the ion bombardment in microdischarges with cylindrical hollow cathode and to suggest possible ways of extending the device lifetime, particle-in-cell/Monte Carlo collision $^{18}$ (PIC/MCC) simulation results of microdischarges with cylindrical hollow cathode operating in argon at 10 and 300 Torr are presented. Discharge characteristics obtained with different electron emission models are compared and the results are interpreted in terms of the device lifetime. Compared to conventional fluid simulations, the simulation technique employed here (PIC/MCC) is capable of capturing nonlocal kinetics and non-Maxwellian electron energy distribution functions often encountered in nonequilibrium discharges. ${ }^{19-21}$ More importantly, PIC/MCC can collect the energy and angle distribution of the ions impinging on the cathode, allowing for a self-consistent model of the ioninduced cathode erosion.

The rest of the manuscript is divided as follows. A description of the simulation model is presented in Sec. II, and the simulation results are discussed in Sec. III. Finally, conclusions are drawn in Sec. IV.

\section{SIMULATION MODEL}

Microdischarges with cylindrical hollow cathode have been modeled using a two-dimensional (2D-3V) axisymmetric PIC/MCC plasma simulator (APPS). ${ }^{15,22}$ The code assumes azimuthal symmetry and solves for the radial and axial coordinates. A multigrid solver is used to determine the electrostatic field and a leapfrog scheme to integrate the equations of motion. The code incorporates an external circuit and Poisson's equation is solved on a uniform grid using superposition of surface and volume charge densities. Particles in the simulation domain retain their three-dimensional velocity in order to capture kinetics accurately. A large number of particles and a radially weighted filter are used to minimize numerical noise.

For a validation of simulation code, a calculation result was compared with experimental results on the hollow cathode geometry with $50 \mathrm{~mm}$ diameter at the $100 \mathrm{mTorr}$ through private communication with Dr. Valery Godyak (Osram Sylvania, Inc., Beverly, MA). The calculated electron 
energy probability function at the discharge center region agrees with experimental results which shows effective electron temperature $0.46 \mathrm{eV}$ (data are not presented).

In the simulation, electrons and atomic argon ions $\left(\mathrm{Ar}^{+}\right)$ are traced as particles while the distribution of background neutrals is assumed to be uniform and constant. APPS uses the null-collision method for efficient computation of the collision processes. ${ }^{23}$ Electron-neutral collisions accounted for in the model include elastic, excitation, and ionization collisions. For the ions, momentum transfer and charge exchange collisions are considered. Coulomb collisions are currently not implemented in the model. Numerical heating of low energy electrons, however, may partially compensate for physical Coulomb interactions. ${ }^{24}$

Initially, the simulation domain is loaded with a spatially uniform Maxwellian distribution of electrons and ions, a dc voltage or current source is applied and the evolution of the particles is followed until the steady state is reached. In the steady state, the electron energy distribution function is no longer Maxwellian and three electron groups are revealed. ${ }^{15}$

A device consists of a hollow cylinder and a circular disk (see Fig. 1). The cylinder has one of its ends closed and acts as the hollow cathode. The diameter of the cathode is $100 \mu \mathrm{m}$ and its length is $150 \mu \mathrm{m}$. The cathode is grounded while the anode disk, which is placed $50 \mu \mathrm{m}$ apart from the cathode, is connected to the power supply. 10 and 300 Torr pressure were considered. When the $p D$ value, where $p$ is pressure and $D$ is the diameter of the hollow cathode, is in the range 0.1-10 Torr $\mathrm{cm}$ and the $V-I$ characteristic shows a negative differential resistance, the discharges are called in the hollow cathode mode. ${ }^{9}$ In this case, the electrons experience oscillatory motion which is due to the strong radial electric field in the hollow cathode region. According to the $p D$ value and electron motions, while 10 Torr discharge belonged to the hollow cathode mode, the 300 Torr case did not.

As an dc discharge, microdischarges with cylindrical hollow cathode rely on the emission of secondary electrons from the cathode surface. ${ }^{25,26}$ Unless otherwise noted, we will assume an effective ion-induced secondary electron emission coefficient $(\gamma)$ of 0.2 , i.e., $\gamma=\Gamma_{e} / \Gamma_{i}$, where $\Gamma_{e}$ and $\Gamma_{i}$ are the emitted electron flux and the incident ion flux, respectively. This effective $\gamma$ accounts for electron emissions due to the bombardment of the cathode by energetic particles and photons, and it is commonly used in computational models. In addition to this electron emission mechanism, electrons can also be emitted under the presence of a strong electric field, i.e., field emissions can take place when the electric field on the cathode region becomes sufficiently large. ${ }^{27}$ The electric field threshold for the onset of field emission from metal surfaces is typically on the order of $\sim 10^{9} \mathrm{~V} / \mathrm{m}$. For surfaces coated with carbon nanotubes (CNT) films, however, the threshold reduces to $\sim 10^{6} \mathrm{~V} / \mathrm{m},{ }^{28}$ which can be easily reached in microdischarges with hollow cathode structure. In this work, we incorporate the field emission process in the simulation by modeling the field emission current with the Fowler-Nordheim equation, ${ }^{29-31}$ i.e., the current is assumed to depend exponentially on the value of the electric field. The constants in the Fowler- (a)

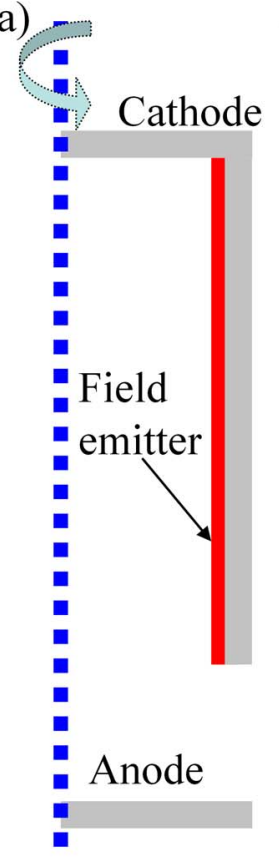

(b)

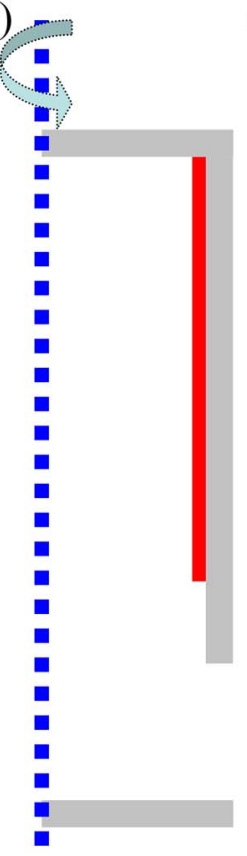

(c)

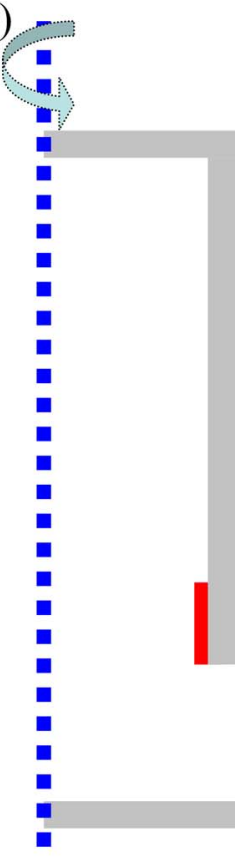

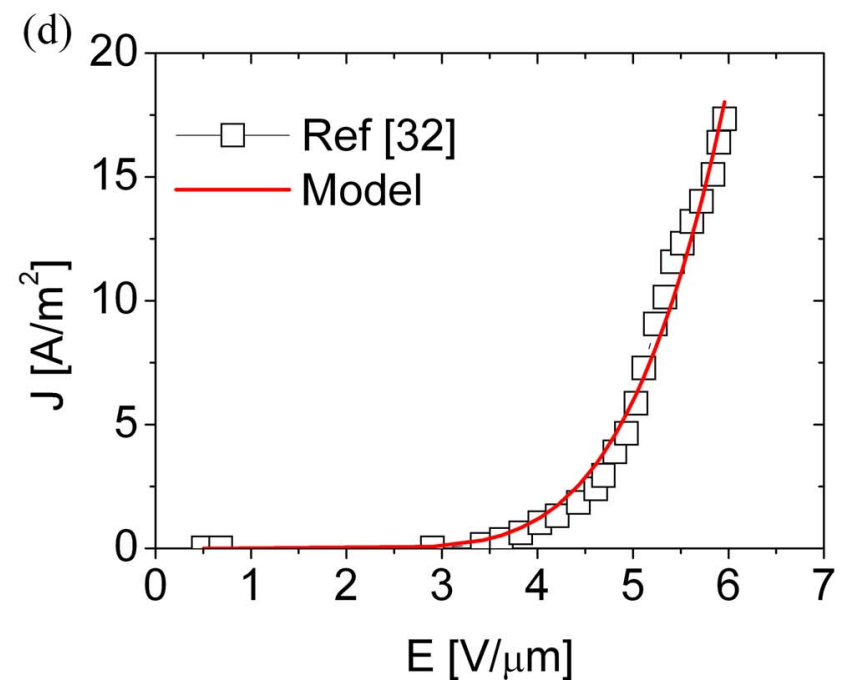

FIG. 1. (Color online) Schematic of the microhollow cathode with three different emission models: (a) Field emitters (CNT film) deposited along the entire cathode region. (b) Field emitters deposited along the entire cathode region but degraded at the entrance of the cathode after operation. (c) Field emitters deposited only at the entrance of the cathode. (d) Field emission current density as a function of the electric field (Ref. 32).

Nordheim equation were obtained by fitting experimental data of the emission properties of a CNT film ${ }^{32}$ [see Fig. 1(d)].

In a real device, field emitters may not be uniformly distributed along the surface of the cathode due to difficulties during the fabrication process and/or the degradation of the emitters by energetic particles during the device operation. Therefore, three scenarios are considered in this study: no field emitter film (baseline for comparison), field emitter film covering the hollow cathode uniformly [Fig. 1(a)], field emitter film degraded at the entrance of the hollow cathode [Fig. 1(b)]. In addition, a case of an idealized emitter located at the entrance of the cathode [Fig. 1(c)] has also been considered. This emitter is assumed to be capable of injecting a 


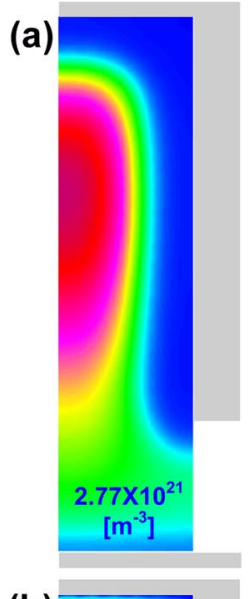

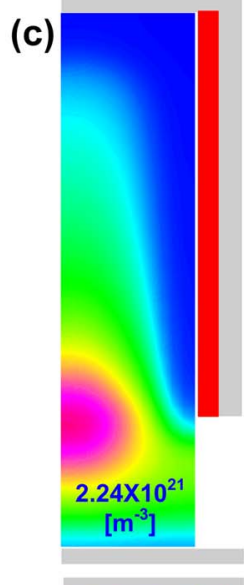

(e)
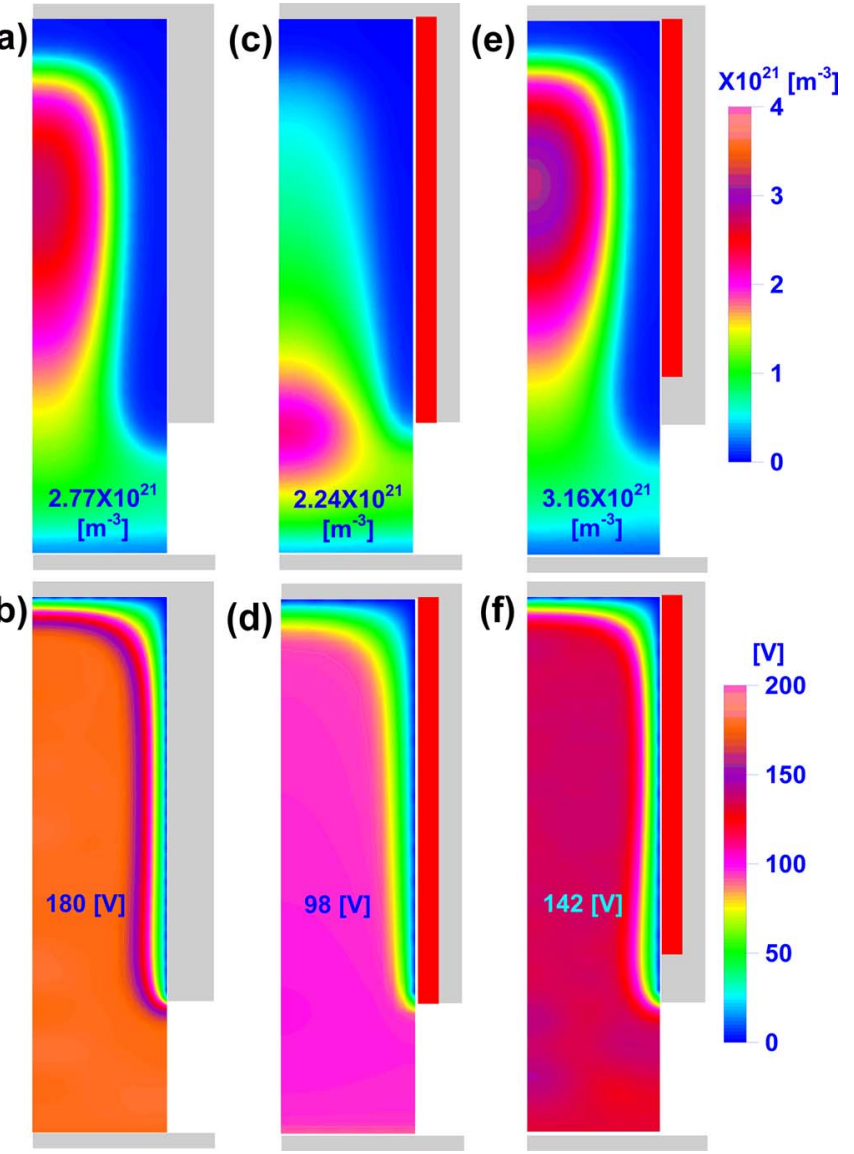

(d)

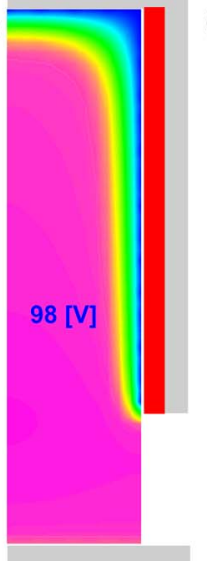

(f)

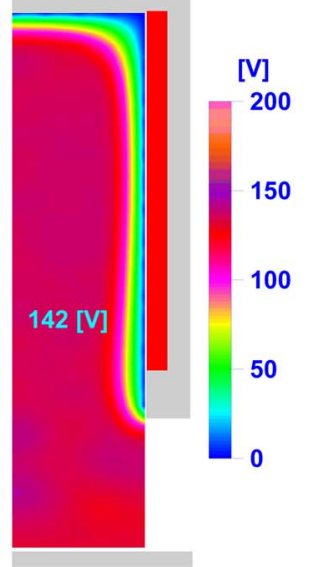

FIG. 2. (Color online) $\mathrm{Ar}^{+}$ion density and potential profiles at 10 Torr $5 \mathrm{~mA}$ : [(a), (b)] without field emitters, [(c), (d)] with field emitters along the entire cathode [Fig. 1(a)], and [(e), (f)] with field emitters only inside the cathode [Fig. 1(b)]. The numbers on each figure indicate the maximum value.

large current density and from a practical point of view it could be realized as a result of the injection of an auxiliary electron beam, photoionization, and/or thermionic emission. ${ }^{33-36}$

Finally, the erosion profile of the cathode is estimated by combining the energy and angle distribution of ions reaching the cathode (PIC-MCC simulation) with the energy- and angle-dependent sputtering yield obtained with SRIM, a group of programs developed by James F. Ziegler that calculate the stopping and range of ions into matter using a quantum mechanical treatment of ion-atom collisions. ${ }^{37,38}$

\section{RESULTS AND DISCUSSION}

Figure 2 shows the density and potential profiles of a 10 Torr $5 \mathrm{~mA}$ argon discharge for three different emission boundary conditions on the cathode. Figures 2(a) and 2(b) correspond to the case without field emitters, Figs. 2(c) and 2(d) to the case with field emitters deposited along the whole cathode surface [as depicted in Fig. 1(a)], and Figs. 2(e) and 2(f) to the case of field emitters covering the surface only deep inside the hollow structure [as depicted in Fig. 1(b)]. For the three cases, while the discharge voltage was changed from 181 through 98 to $142 \mathrm{~V}$, the maximum plasma density remains within the same order of magnitude: $\sim 2 \times 10^{21} \mathrm{~m}^{-3}$. The spatial distribution of the plasma, however, strongly depends on the boundary condition. In the absence of field emitters [Fig. 2(a)], the plasma density is maximum well inside the hollow cathode. In the 10 Torr case, the discharge operates in the hollow cathode mode with a virtual anode extending along the axis of the hollow cathode [Fig. 2(b)]. Secondary electrons are accelerated in the radial direction in the cathode sheath and acquire a pendular motion. ${ }^{13}$ When the cathode is uniformly coated with a film of field emitters, the peak plasma density is reached at the entrance of the cathode [Fig. 2(c)] and the cathode sheath potential is reduced from $181 \mathrm{~V}$ to $98 \mathrm{~V}$ [compare Figs. 2(b) and 2(d)]. The displacement of the plasma towards the entrance of the cathode [compare Figs. 2(a) and 2(c)] is due to the local enhancement of the electric field around the edge of the cathode. Although in the simulation the properties of the field emitters are the same independently of their location on the cathode, the self-consistent enhancement of the electric field at the entrance of the cathode drives most current through that region. As it will be discussed later, the edge of the cathode is susceptible to energetic ion bombardment, and as a result one would expect the field emitters in that area to get degraded quickly. Once this damage has taken place, one would reach a situation similar to the third case [Figs. 2(e) and 2(f)]. In this case, the peak plasma density gets back inside the hollow cathode and thanks to the contributions of the field emitters a denser plasma and a lower sheath potential are obtained [compare Figs. 2(a) and 2(b) with Figs. 2(e) and 2(f)]. The reduced sheath potential when the cathode is coated with a CNT film has also been observed experimentally by Eden and Park. ${ }^{39}$

The lowering of the operation voltage due to the field emitters can be analyzed qualitatively with a simple analytical model. The current density at the cathode $\left(J_{T}\right)$ is given by $J_{T}=J_{i}+J_{\gamma}+J_{\text {others }}$, where $J_{i}$ is the ion current density, $J_{\gamma}$ $=\gamma J_{i}$ is the effective ion-induced secondary electron emission current density, and $J_{\text {others }}$ is the current density due to other electron emission processes, in this case field emission.

Approximating the ion current to the cathode with the Child-Law model, ${ }^{40}$ the ion current is related to the applied voltage as

$$
J_{i}=\frac{4}{9} \varepsilon_{0}\left(\frac{2 e}{M}\right)^{1 / 2} \frac{V^{3 / 2}}{s^{2}} .
$$

As a result, the applied voltage is given by $V$ $=V_{o}(1-\alpha)^{2 / 3}$, where $\alpha=J_{\text {others }} / J_{T}$ is the ratio of field emission current to the total current, and $V_{o}$ is the voltage required to sustain the discharge in the absence of field emitted current $\left(J_{\text {others }}=0\right)$,

$$
V_{0}=\left[\frac{J_{T}}{1+\gamma} \frac{9}{4}\left(\frac{M}{2 e}\right)^{1 / 2} \frac{s^{2}}{\varepsilon_{0}}\right]^{2 / 3} .
$$

Figure 3 compares the applied voltage dependence on the 


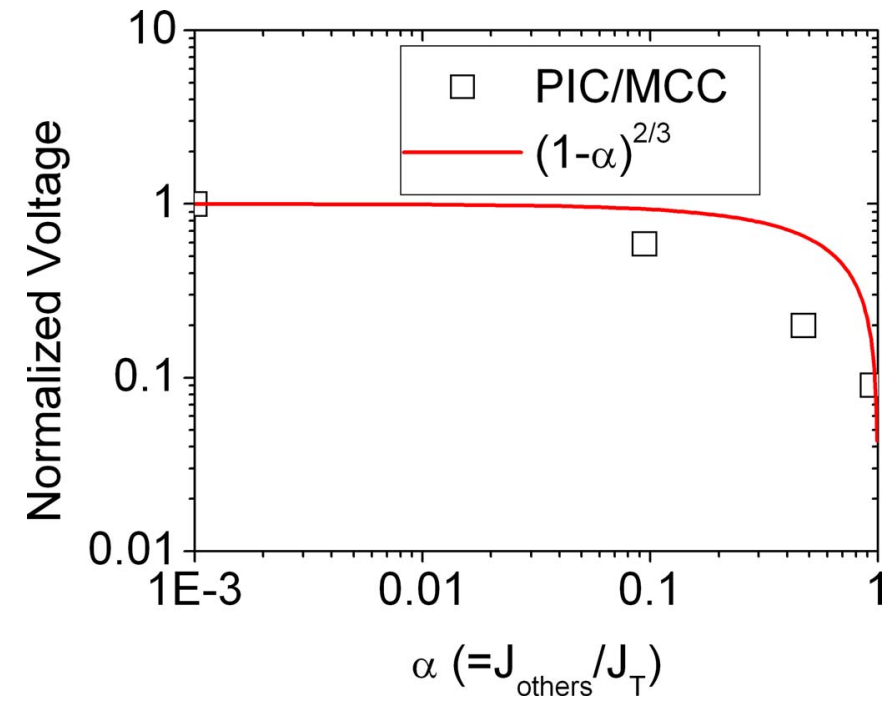

FIG. 3. (Color online) Cathode sheath potential as a function of emission current $(\alpha)$ for a 10 Torr $5 \mathrm{~mA}$ argon discharge.

ratio of field emitted to total current $(\alpha)$ predicted by the simple analytical model and the PIC simulations. The analytical model underestimates the voltage reduction due to the extrapolation of the Child-Law model to a collisional sheath in the presence of strong electron emission. Nonetheless, the model captures the qualitative behavior.

One of the main drawbacks of hollow cathode devices is their short lifetime. The lifetime of microhollow cathode devices is limited by the erosion of the cathode surface. Assuming that the erosion is mainly due to ion impact, the erosion profile can be predicted by combining the ion energy and angle distributions on the cathode surface obtained from the PIC simulations with the sputtering yield calculated with SRIM. The erosion profile $[e(z)]$ is then given by

$$
e(z)=\int_{0}^{\infty} \int_{0}^{\pi / 2} Y(\varepsilon, \theta) f(\varepsilon, \theta, z) d \theta d \varepsilon
$$

where $Y(\varepsilon, \theta)$ and $f(\varepsilon, \theta, z)$ are the sputtering yield and the ion energy-angle distribution on the cathode surface. It is clear that the erosion profile depends on the material properties of the cathode as well as on the discharge characteristics. For a given cathode material, $Y(\varepsilon, \theta)$ is fixed and the only way to control the lifetime of the device is by adjusting the ion flux onto the cathode surface $[f(\varepsilon, \theta, z)]$.

In general, the sputtering yield (Fig. 4) increases monotonically with the energy of the incident ion and is higher for particles striking perpendicularly onto the cathode surface. As a result, a decrease on the applied potential and an increase on the number of collisions ions undergo as they transit the sheath will result in an improved lifetime of the device. The former can be achieved by incorporating field emitters and the latter by operating at high pressure. Figure 5 compares the average kinetic energy of $\mathrm{Ar}^{+}$ions at 10 and 300 Torr. At 10 Torr, the maximum average ion kinetic energy is $\sim 76 \mathrm{eV}$, nearly half the applied voltage. At 300 Torr,

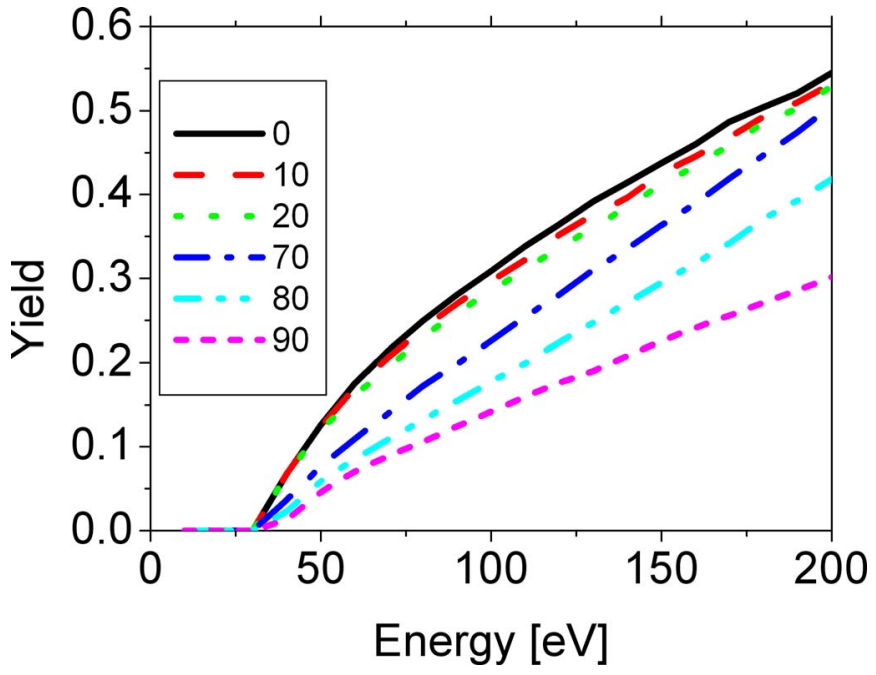

FIG. 4. (Color online) Sputtering yield of Mo by argon ions obtained with SRIM (Refs. 37 and 38). Angle measured with respect to a perpendicular to the surface.

however, the maximum average ion kinetic energy reduces to $\sim 1.4 \mathrm{eV}(\sim$ a tenth of the applied voltage) due to frequent collisions in the sheath. In addition, collisions in the sheath also favors the device lifetime by reducing the number of perpendicular impacts on the cathode (see Fig. 4 for the incidence angle dependence of the sputtering yield for $\mathrm{Ar}^{+}$on Mo).

Figure 6 shows the actual energy and angle distributions of the ions bombarding the cathode at three different locations. The locations are marked as (1), (2), and (3), and they are graphically shown in Fig. 7. The dotted lines in Fig. 6

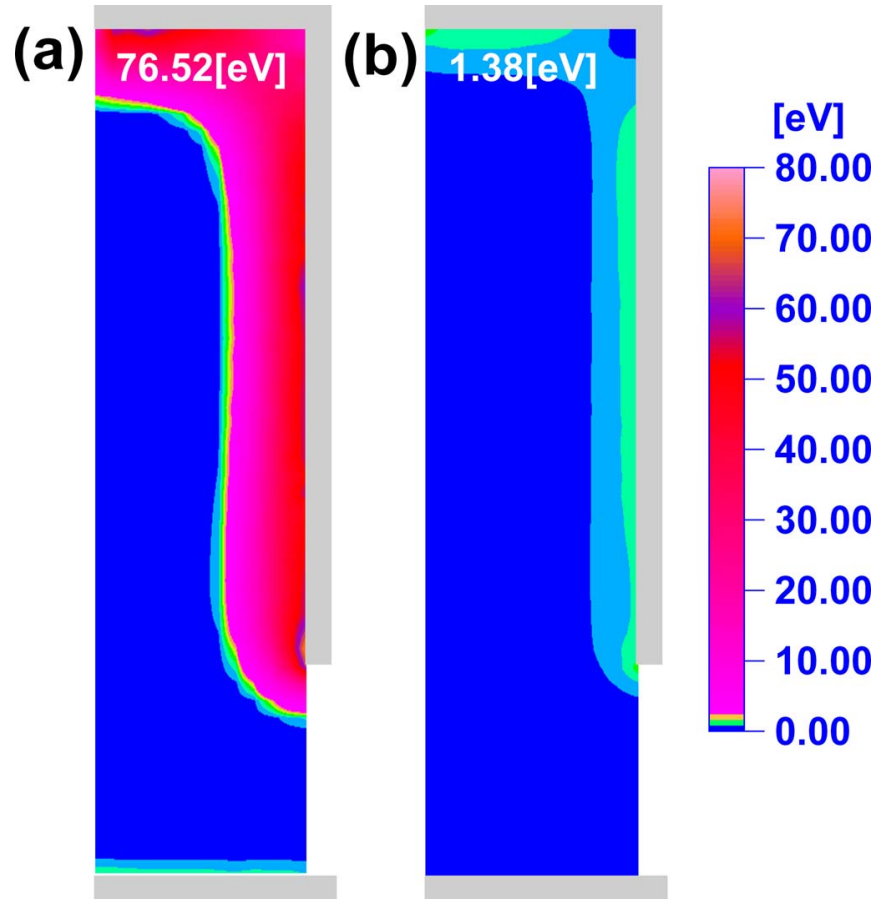

FIG. 5. (Color online) Average ion kinetic energy profile for argon discharges at (a) 10 Torr $5 \mathrm{~mA}$, and (b) 300 Torr $5 \mathrm{~mA}$. The numbers on each figure indicate the maximum value. 


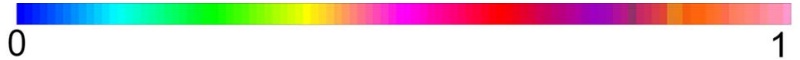

(a)
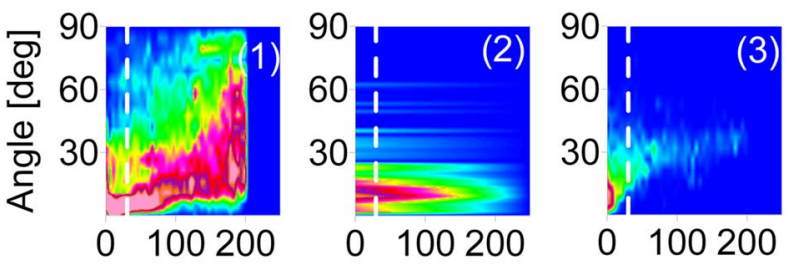

Energy $[\mathrm{eV}]$

(b)
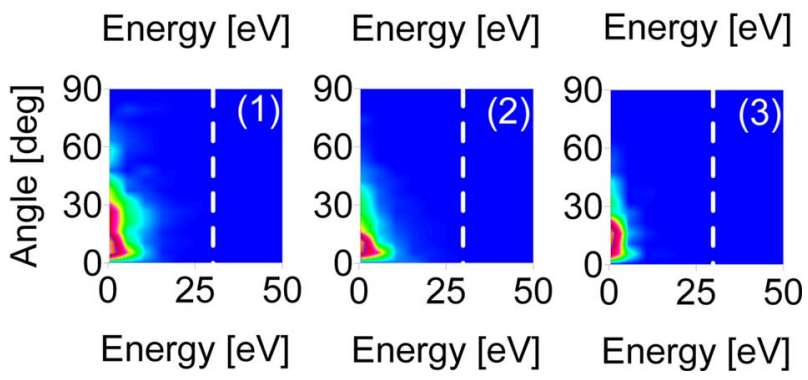

FIG. 6. (Color online) Energy and angle distribution of $\mathrm{Ar}^{+}$ions at several locations on the cathode surface (a) 10 Torr $5 \mathrm{~mA}$ and (b) 300 Torr $5 \mathrm{~mA}$. The dashed lines indicate the sputtering threshold energy.

(a)

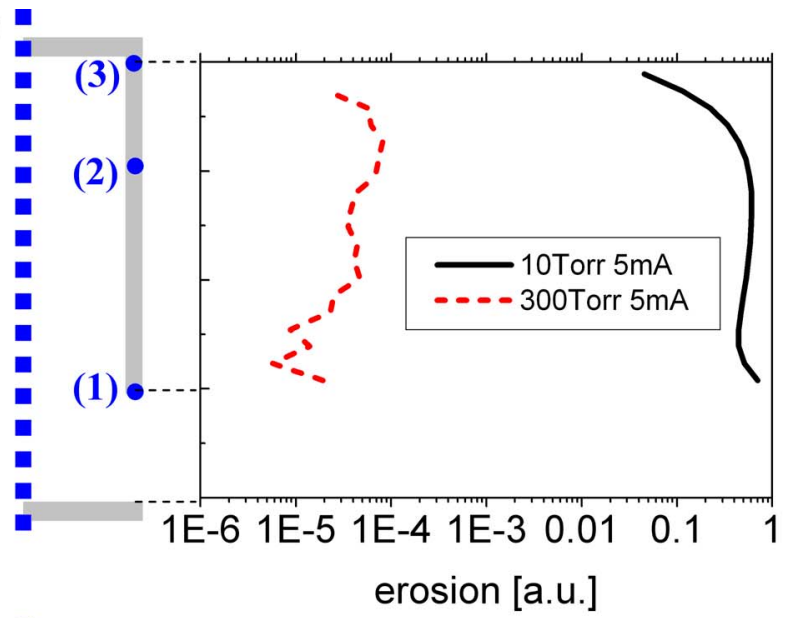

(b)

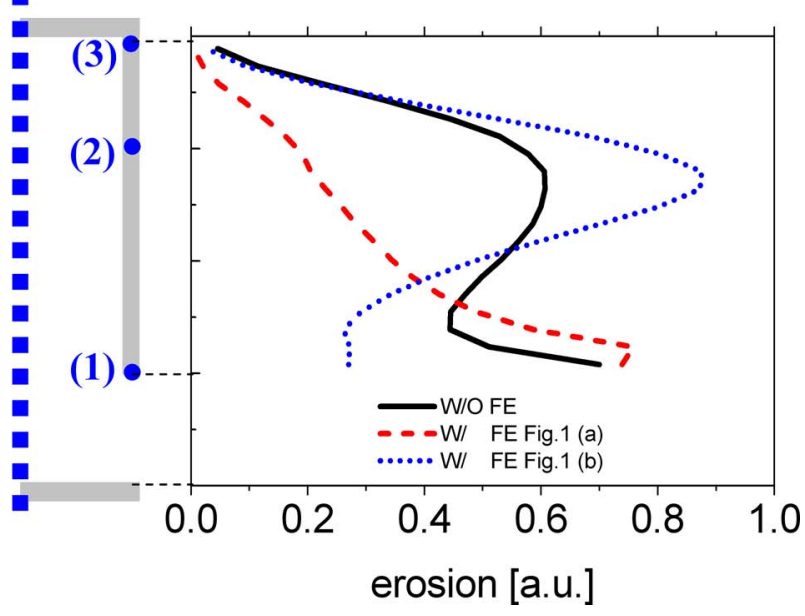

FIG. 7. (Color online) Erosion profiles. (a) Influence of the operating pressure in the absence of field emission. (b) Influence of the field emission for 10 Torr $5 \mathrm{~mA}$ discharges. indicate the sputtering threshold for Mo by Ar ions $(\sim 27 \mathrm{eV}$ as shown in Fig. 4). At 10 Torr the energy distribution of the $\mathrm{Ar}^{+}$ions striking on the cathode extends beyond the sputtering threshold. At 300 Torr, however, most ions arrive at the cathode with energies below the sputtering threshold. As a result, the sputter damage of the cathode is largely reduced by operating at 300 Torr [Fig. 7(a)]. At 10 Torr and in the absence of field emission, the sputtering takes place most significantly at the entrance of the cathode and inside at the center [Fig. 7(b)]. On the other hand, for the case in which field emitters have been deposited along the whole cathode surface, the erosion is maximum at the entrance of the discharge. These are the regions that receive the highest influx of particles. It is noted, however, that the sputtering profile is not proportional to the net ion flux because the sputtering yield depends on the angle of incidence (Fig. 4) and the angular distribution at the entrance of the cathode and inside the cathode are quite different [Fig. 6(a)]. It is interesting to note that when field emitters are deposited only inside the cathode, the sputtering at the entrance reduces as a result of the concentration of the discharge near the regions where field emission takes place [Figs. 2(a) and 2(e)]. In that region, however, the sputtering is enhanced as a result of the increased flux (Fig. 7).

Ideally one would like to operate the discharge with an applied voltage that is below the sputtering yield of the cathode material (tens of volts) and yet above the ionization threshold of the background gas so that no sputter damage is caused by the ions bombarding the cathode and secondary electrons still can gain enough energy to ionize the background gas. In Fig. 8, the potential profiles of three discharges in which an idealized emitting mechanism is incorporated at the entrance of the cathode [Fig. 1(c)] at 300 Torr are presented. For a drastic reduction of the applied potential down to tens of volts, the injected current needs to be comparable to the net discharge current, which for the geometry of Fig. 1(c) requires a current density of $100 \mathrm{~A} / \mathrm{cm}^{2}$. Such a high current density is likely to trigger the onset of thermionic emission which is currently not considered in the model.

\section{SUMMARY}

In microdischarges with hollow cathode structure, high electric fields are generated due to the reduced dimensions of the devices and this high electric field can trigger field emission from the cathode surface with changes in the discharge characteristics. Indeed, the onset of field emission leads to a reduction of the discharge voltage and changes the density profile. By combining the sputtering yield of the cathode material with the self-consistent energy and angle distribution of ions impinging onto the cathode, the erosion profile of the cathode can be predicted. In agreement with experiments, the simulations suggest an extended lifetime when the discharges operate at high pressure. This is as a result of the larger number of collisions the ions undergo as they transit the sheath, which leads to a reduction of their final energy and a broader angular distribution. Further improvements can be reached by the reduction of the operating voltage due 


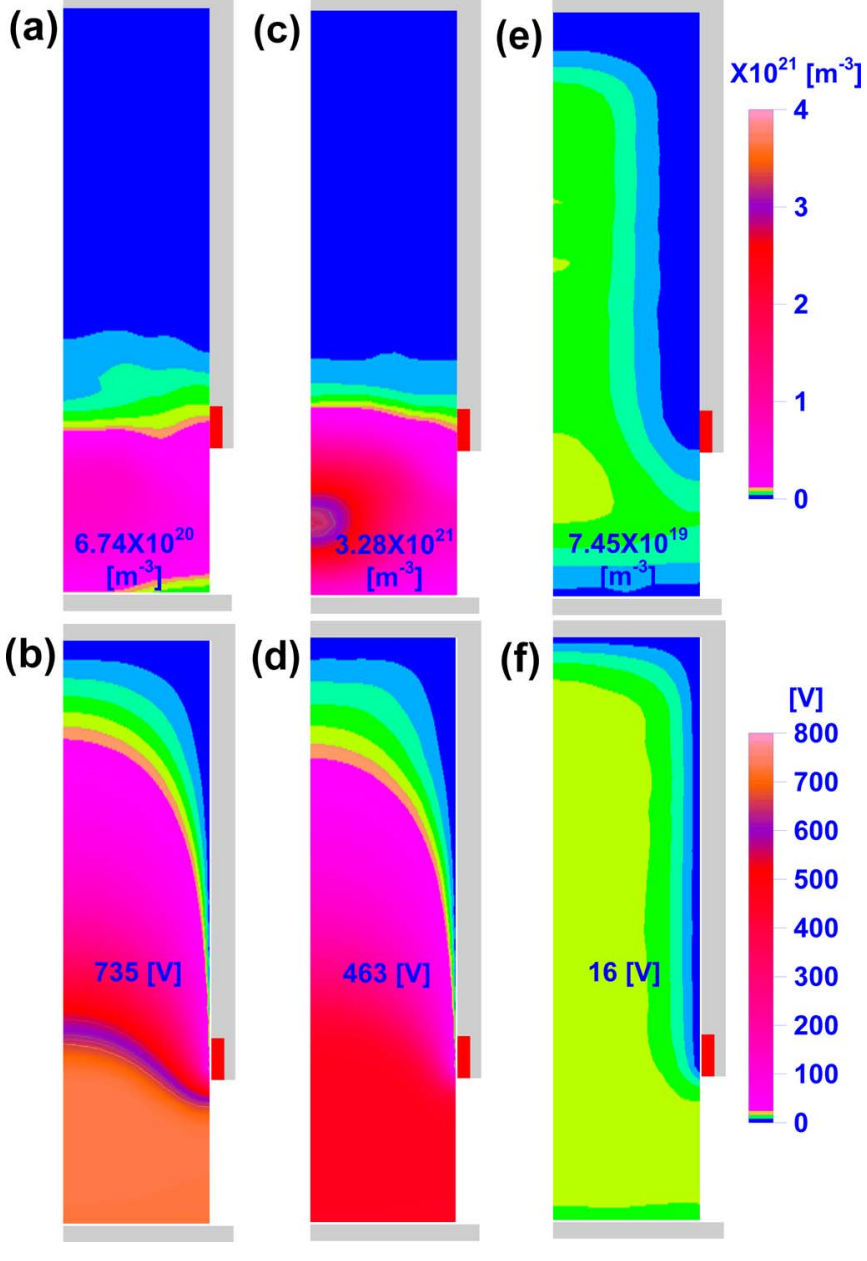

FIG. 8. (Color online) $\mathrm{Ar}^{+}$ion density and potential profiles at 300 Torr $0.5 \mathrm{~mA}$ for the geometry shown in Fig. 1(c). Emitter current density of (a, b) $j=1 \mathrm{~A} / \mathrm{cm}^{2},(\mathrm{c}, \mathrm{d}) j=10 \mathrm{~A} / \mathrm{cm}^{2}$, and (e, f) $j=100 \mathrm{~A} / \mathrm{cm}^{2}$. The numbers on each figure indicate the maximum value.

to the incorporation of field emitters (e.g., carbon nanotubes films) on the cathode surface. For realistic field emitters, however, the reduction in the discharge voltage is not large enough as to bring the operating voltage below the sputtering yield of the cathode material. As a result, one is to expect the improvements brought in by incorporating field emitters to degrade with time as a result of the physical erosion of the emitters. Combining different electron emitting physical mechanisms (e.g., field emission, photoionization, thermionic emission, ...), one could reach a scenario in which the operating voltage is lower than the sputtering yield of the cathode material and yet greater than the ionization threshold of the background gas. Under these conditions, the lifetime of the microhollow cathode could be drastically extended.

\section{ACKNOWLEDGMENTS}

This work was supported by the Korea Science and Engineering Foundation (KOSEF) under Grant No. R01-2007000-10730-0.
${ }^{1}$ K. H. Schoenbach, R. Verhappen, T. Tessnow, and R. E. Peterkin, Appl. Phys. Lett. 68, 13 (1995).

${ }^{2}$ K. H. Schoenbach, A. El-Habachi, W. Shi, and M. Ciocca, Plasma Sources Sci. Technol. 6, 468 (1997).

${ }^{3}$ J. Hopwood, Ionized Physical Vapor Deposition, Thin Film Series (Academic, San Diego, 2000), Vol. 27.

${ }^{4}$ J. Engemann, Surf. Coat. Technol. 169-170, 14 (2003).

${ }^{5}$ C. Jiang, A. Kuthi, and M. A. Gundersen, Appl. Phys. Lett. 87, 131501 (2005).

${ }^{6}$ M. Miclea, K. Kunze, J. Franzke, and K. Niemax, Spectrochim. Acta, Part B 57, 1585 (2002).

${ }^{7}$ C. Jiang, A. Kuthi, and M. A. Gundersen, Appl. Phys. Lett. 86, 024105 (2005).

${ }^{8}$ S.-J. Park, J. D. Readle, A. Y. Chang, L. Z. Hua, K. S. Kim, and J. G. Eden, in Proceedings of the IEEE 33rd International Conference on Plasma Science (ICOPS), Traverse City, MI, 2006, p. 360.

${ }^{9}$ K. H. Becker, K. H. Schoenbach, and J. G. Eden, J. Phys. D 39, R55 (2006).

${ }^{10}$ K. H. Becker, U. Kogeschatz, K. H. Schoenbach, and R. J. Barker, Nonequilibrium Air Plasmas at Atmospheric Pressure (Institute of Physics, Bristol, 2004), Chap. 6.5.

${ }^{11}$ F. Iza, G. J. Kim, S. M. Lee, J. K. Lee, J. L. Walsh, Y. T. Zhang, and M. G. Kong, Plasma Processes Polym. 5, 322 (2008).

${ }^{12}$ K. A. Peard, R. C. Tobin, K. Rozsa, and Z. Donko, IEEE J. Quantum Electron. 30, 1181 (1994).

${ }^{13}$ V. I. Kolobov and L. D. Tsendin, Plasma Sources Sci. Technol. 4, 551 (1995).

${ }^{14}$ Z. Donko, Z. Naturforsch., A: Phys. Sci. 48, 457 (1993).

${ }^{15}$ G. J. Kim, F. Iza, and J. K. Lee, J. Phys. D 39, 4386 (2006).

${ }^{16}$ J. P. Boeuf, L. C. Pitchford, and K. H. Schoenbach, Appl. Phys. Lett. 86, 071501 (2005).

${ }^{17}$ F. Iza, J. K. Lee, and M. G. Kong, Phys. Rev. Lett. 99, 075004 (2007).

${ }^{18}$ C. K. Birdsall and A. B. Langdon, Plasma Physics via Computer Simulation (Adam Hilger, Bristol, 1991).

${ }^{19}$ H. C. Kim and J. K. Lee, Phys. Rev. Lett. 93, 085003 (2004).

${ }^{20}$ G. Y. Park, S. J. You, F. Iza, and J. K. Lee, Phys. Rev. Lett. 98, 085003 (2007).

${ }^{21}$ H. C. Kim, F. Iza, S. S. Yang, M. R. Radjenovic, and J. K. Lee, J. Phys. D 38, R283 (2005).

${ }^{22}$ F. Iza and J. K. Lee, Comput. Phys. Commun. 177, 72 (2007).

${ }^{23}$ V. Vahedi and M. Surendra, Comput. Phys. Commun. 87, 179 (1995).

${ }^{24}$ M. Turner, Phys. Plasmas 13, 033506 (2006).

${ }^{25}$ E. H. Choi, H. J. Oh, Y. G. K, J. J. Ko, J. Y. Lim, J. G. Kim, D. I. Kim, G. Cho, and S. O. Kang, Jpn. J. Appl. Phys., Part 1 37, 7015 (1998).

${ }^{26}$ A. V. Phelps and Z. L. Petrovic, Plasma Sources Sci. Technol. 8, R21 (1999).

${ }^{27}$ R. Gomer, Field Emission and Field Ionization (Springer, Berlin, 1993).

${ }^{28}$ J. M. Bonard, H. Kind, T. Stockli, and L. O. Nilsson, Solid-State Electron. 45, 893 (2001).

${ }^{29}$ R. H. Fowler and L. W. Nordheim, Proc. R. Soc. London A119, 173 (1928).

${ }^{30}$ S.-J. Park, J. G. Eden, and K.-H. Park, Electron. Lett. 40, 563 (2004).

${ }^{31}$ J. M. Bonard, N. Weiss, H. Kind, T. Stockli, L. Forro, K. Kern, and A. Chatelain, Adv. Mater. (Weinheim, Ger.) 13, 184 (2001).

${ }^{32}$ S. J. Kyung, M. Vornoko, Y. H. Lee, C. W. Kim, J. H. Lee, and G. Y. Yeom, Surf. Coat. Technol. 201, 5378 (2007).

${ }^{33}$ W. Hartmann, V. Dominic, G. F. Kirkman, and M. A. Gundersen, Appl. Phys. Lett. 53, 1699 (1988).

${ }^{34}$ W. Hartmann, V. Dominic, G. F. Kirkman, and M. A. Gundersen, J. Appl. Phys. 65, 4388 (1989).

${ }^{35}$ A. Anders, S. Anders, and M. A. Gundersen, Phys. Rev. Lett. 71, 364 (1993).

${ }^{36}$ A. Anders, S. Anders, and M. A. Gundersen, J. Appl. Phys. 76, 1494 (1994).

${ }^{37}$ J. F. Ziegler, J. P. Biersack, and M. D. Ziegler, SRIM: The Stopping and Range of Ions in Matter (Lulu, Chester, 2008).

${ }^{38}$ SRIM software can be found at the http://www.srim. org.

${ }^{39}$ J. G. Eden and S. J. Park, Phys. Plasmas 13, 057101 (2006).

${ }^{40}$ M. A. Lieberman and A. J. Lichtenberg, Principles of Plasma Discharges and Materials Processing (Wiley, New Jersey, 2005). 